\title{
BMJ open Mental health status and risk of new cardiovascular events or death in patients with myocardial infarction: a population-based cohort study
}

To cite: Nielsen TJ, Vestergaard $\mathrm{M}$, Christensen B, et al. Mental health status and risk of new cardiovascular events or death in patients with myocardial infarction: a population-based cohort study. BMJ Open 2013;3: e003045. doi:10.1136/ bmjopen-2013-003045

- Prepublication history and additional material for this paper is available online. To view these files please visit the journal online (http://dx.doi.org/10.1136/ bmjopen-2013-003045).

Received 12 April 2013 Revised 3 July 2013 Accepted 4 July 2013

${ }^{1}$ Section for General Medical Practice, Aarhus University, Aarhus C, Denmark

${ }^{2}$ Section for General Medical Practice and Research Unit for General Practice, Aarhus University, Aarhus C,

Denmark

${ }^{3}$ Research Unit for General Practice, Aarhus University, Aarhus C, Denmark

Correspondence to Tine Jepsen Nielsen; tjn@folkesundhed.au.dk

\section{ABSTRACT}

Objective: To examine the association between mental health status after first-time myocardial infarction (MI) and new cardiovascular events or death, taking into account depression and anxiety as well as clinical, sociodemographic and behavioural risk factors. Design: Population-based cohort study based on questionnaires and nationwide registries. Mental health status was assessed 3 months after MI using the Mental Component Summary score from the Short-Form 12 V.2.

Setting: Central Denmark Region.

Participants: All patients hospitalised with first-time MI from 1 January 2009 through 31 December 2009 $(\mathrm{n}=880)$. The participants were categorised in quartiles according to the level of mental health status (first quartile=lowest mental health status).

Main outcome measures: Composite endpoint of new cardiovascular events (MI, heart failure, stroke/transient ischaemic attack) and all-cause mortality.

Results: During 1940 person-years of follow-up, 277 persons experienced a new cardiovascular event or died. The cumulative incidence following 3 years after $\mathrm{MI}$ increased consistently with decreasing mental health status and was $15 \%(95 \% \mathrm{Cl} 10.8 \%$ to $20.5 \%)$ for persons in the fourth quartile, $29.1 \%(23.5 \%$ to $35.6 \%)$ in the third quartile, $37.0 \%$ (30.9\% to $43.9 \%)$ in the second quartile, and $47.5 \%$ (40.9\% to $54.5 \%$ ) in the first quartile. The HRs were high, even after adjustments for age, sociodemographic characteristics, cardiac disease severity, comorbidity, secondary prophylactic medication, smoking status, physical activity, depression and anxiety $\left(\mathrm{HR}_{3 \mathrm{rd}}\right.$ quartile 1.90 (95\% $\mathrm{Cl} 1.23$ to 2.93$), \mathrm{HR}_{2 \text { nd quartile }} 2.14$ (1.37 to 3.33), $\mathrm{HR}_{1 \text { st quartile }} 2.23$ (1.35 to 3.68 ) when using the fourth quartile as reference).

Conclusions: Low mental health status following firsttime MI was independently associated with an increased risk of new cardiovascular events or death. Further research is needed to disentangle the pathways that link mental health status following MI to prognosis and to identify interventions that can improve mental health status and prognosis.

\section{ARTICLE SUMMARY}

Article focus

- Myocardial infarction (MI) is often followed by mental health problems such as depression, anxiety and low mental health status.

- Mounting evidence indicates that depression and anxiety after $\mathrm{MI}$ increase the risk of adverse longterm outcome. No previous studies have examined the association between mental health status after $\mathrm{MI}$ and outcome, independent of depression and anxiety.

- This study examines the association between mental health status after first-time $\mathrm{MI}$ and new cardiovascular events or death when taking into account depression and anxiety as well as clinical, sociodemographic and behavioural risk factors.

Key messages

- During the 3 years after $\mathrm{MI}$, patients with the lowest mental health status had an almost $50 \%$ risk of new cardiovascular events or death.

- Low mental health status after MI was a strong predictor of new cardiovascular events or death, independent of depression, anxiety and clinical, sociodemographic and behavioural risk factors.

Strengths and limitations of this study

- The major strengths of this study are its population-based nature and the homogeneous study population. The response rate was reasonably high, and information on outcome was collected without loss to follow-up.

- We were able to take into account important mediators such as depression, anxiety and potential behavioural mediators such as physical activity. However, we cannot rule out the possibility of residual confounding.

\section{INTRODUCTION}

Myocardial infarction (MI) is a severe life event followed by an increased risk of mental health problems such as depression, ${ }^{1}$ anxiety ${ }^{2}$ and low mental health status. ${ }^{3}$ Several studies 
have shown that depression ${ }^{4}$ and anxiety ${ }^{2}$ after MI are associated with a higher risk of cardiovascular events and death, but much less is known about the impact of broader measures of mental health. Mental health status is a generic and broad measure of mental health, which may be useful as a tool to quantify important prognostic aspects of mental health not captured by the more disease-specific measures of depression and anxiety. Four studies $^{5-8}$ have investigated the association between mental health status following MI and prognosis. All these have found that low mental health status was significantly associated with increased risk of adverse outcome, independent of clinical risk factors. However, since none of the former studies adjusted for depression or anxiety, it remains unknown whether mental health status in itself adds unique knowledge about the prognosis.

Our aim was to examine the association between mental health status and new cardiovascular events or death in patients with first-time MI when taking into account depression, anxiety, and clinical, sociodemographic and behavioural risk factors.

\section{METHODS}

We conducted a population-based cohort study comprising people in the Central Denmark Region (1 250000 inhabitants) with a first-time MI based on data from nationwide registers and questionnaires.

\section{Participants}

We consecutively invited all patients discharged from hospital with a first-time MI from 1 January 2009 to 31 December 2009. The establishment of the cohort is described in detail elsewhere. ${ }^{9}$ Data on patients discharged with MI (in accordance with the International Classification of Diseases (ICD-10) code I21) ${ }^{10}$ were received from the Danish National Patient Register on a monthly basis. Patients who had been discharged with MI between 1994 and 2008 were excluded to identify first-time cases. Information on name, address and vital status was obtained from the Civil Registration System, ${ }^{11}$ which also provided the unique personal identification number used to link data between the registers and questionnaires.

\section{Data collection}

A pilot-tested hard-copy questionnaire was sent to all participants 12-14 weeks after their discharge from hospital, and non-responders received two reminders. ${ }^{9}$ The study was approved by the Danish Data Protection Agency (J.nr. 2009-41-3018), the Scientific Research Evaluation Committee of the Danish Academy of General Practitioners (ref. no. 03-2009), and written informed consent was obtained from all participants.

\section{Mental health status}

Mental health status was measured using the Mental Component Summary (MCS) score from the validated
Danish version of the Short-Form 12 V.2 Health Survey (SF-12). ${ }^{12-14}$ SF-12 consists of 12 items, and the MCS score mainly comprises the six mental items ('Vitality', 'Role-Emotional' (2 items), 'Social Function' and 'Mental Health' (2 items)), but the six physical items are also included in the computation. ${ }^{22}$ The SF-12 scores were calculated following the norm-based scoring algorithm ${ }^{12}$ using weights derived from confirmatory factor analysis. ${ }^{15}$ The MCS score is thus linearly transformed in a way that allows comparison with the mean score (50) and the SD (10) in the general US population in $1998 .{ }^{12}$ The MCS has demonstrated good construct validity. ${ }^{15}$ The wording of the mental health status items can be found in the supplemental material (see online supplementary figure 1 ).

\section{Depression and anxiety}

We assessed depression and anxiety symptoms using the Hospital Anxiety and Depression Scale (HADS). ${ }^{16}$ Participants were categorised as having anxiety or depression if they had a score of $\geq 8$ on the HADS-A scale or the HADS-D scale. The HADS was designed to be valid in clinical populations with symptoms of physical disease and hence leaves out items that may be endorsed by physical rather than mental states. ${ }^{16}{ }^{17}$ It has formerly been validated in MI patients ${ }^{18}{ }^{19}$ and has proven to have satisfactory reliability (HADS-A and HADS-D Cronbach's $\alpha \approx 0.80) .{ }^{18}{ }^{20}$ Among MI patients, a HADS-D $\geq 8$ identified possible cases of depression with a sensitivity of $65 \%$ and a specificity of $90 \%$ (compared with a diagnosis of depression based on a Structured Clinical Interview for DSM-IV). ${ }^{19}$ Among acute coronary syndrome patients, a HADS-A $\geq 8$ identified possible cases of anxiety with a sensitivity of $91 \%$ and a specificity of $61 \%$ (compared with a diagnosis of generalised anxiety disorder based on a structured clinical interview for DSM-IV). ${ }^{21}$

\section{Comorbidity and cardiac disease severity}

Information on comorbidity was retrieved from the Danish National Patient Register, ${ }^{22}$ the Danish National Diabetes Register, ${ }^{23}$ and the prescription database covering the entire Central Denmark Region. ${ }^{24}$ The Danish National Patient Register provided information on stroke (ICD-10: I61, I63, I64), transient cerebral ischaemic attack (ICD-10: DG45, DG46), heart failure (ICD-10: I11.0, I13.0, I13.2, I42.0, I42.6, I42.7, I42.9, I50.0, I50.1, I50.9), and revascularisation (ICD-10: KFN, KFW) from 1994 to 2008. The Danish National Diabetes Register provided information on diabetes mellitus from 1990 to 2008 according to an algorithm developed on the basis of information from four nationwide registers. ${ }^{23}$ The prescription database provided information on all reimbursed drugs according to the Anatomical Therapeutic Chemical Classification System (ATC), dispensing dates and the total number of tablets dispensed. Participants were categorised with hypertension if they had redeemed prescriptions for at least two classes of 
antihypertensive drugs (ATC: C02A-D, C02 L, C03A-B, C03D-E, C03X, C04, C05, C07, C08, C09) 0-180 days before the index MI. Participants were categorised with depression before MI if they had redeemed a prescription for an antidepressant (ATC: N06A) 0-180 days before the index MI. Participants were categorised with severe mental disorder if they had redeemed a prescription for antipsychotics (ATC: N05A) 0-180 days before the index MI.

Cardiac disease severity was measured by the British Medical Research Council (MRC) dyspnoea score, a selfreport instrument. ${ }^{25} \mathrm{~A}$ score $\geq 3$ has been shown to provide a simple and valid method for predicting overall mortality. $^{26}$

\section{Health behaviour, healthcare interventions and sociodemographics}

Data on smoking, alcohol use, physical activity, intake of fruit and vegetables, intake of fish, intake of fish oil supplement, height and weight (body mass index=weight $(\mathrm{kg})$ per height $\left(\mathrm{m}^{2}\right)$ ) were self-reported and classified according to the general recommendations from the Danish National Board of Health. ${ }^{9}$ To assess physical activity, we asked, "How many days per week are you generally physically active for at least 30 minutes per day? You may include any physical activity at work or in your spare time that makes your pulse rate increase". Response options were from zero days to every day per week. Physical activity was computed as a continuous variable (days/week).

We defined cardiac rehabilitation ${ }^{27} 28$ in the questionnaire and asked whether they had participated in hospital-based phase two cardiac rehabilitation. Those who responded "yes, and I took part" were classified as 'participants', whereas those who responded "yes, but I didn't take part" or "no" were classified as "non-participants'. 9

Drug prescription data were obtained from the prescription database. ${ }^{24}$ Data on aspirin (ATC: B01AC06), clopidogrel (ATC: B01AC04), statins (ATC: C10AA), $\beta$-blockers (ATC: C07), ACE-inhibitors/angiotensin 2 receptor blockers (ATC: C09), furosemide (ATC: C03C), aldosterone antagonists (ATC: C03D) and antidepressants (ATC: N06A) were collected. We calculated whether the participant had tablets available on the day that we sent the questionnaire (the number of tablets on the last redeemed prescription before the questionnaire was sent $\geq$ the number of days to the questionnaire was sent) and defined the participant as 'receiving treatment' if tablets were available. We defined the participant as 'receiving secondary prophylactic medication' if the participant was receiving treatment with three or more of the following drugs: aspirin, clopidogrel, statins and $\beta$-blockers. We defined the participant as 'receiving heart failure medication' if the participant was receiving treatment with furosemide or aldosterone antagonists.

Data on age at MI and sex were obtained from the Civil Registration System. ${ }^{11}$ Each participant's sociodemographic characteristics (cohabitation status, education, labour market status) from the year before MI (2008) were retrieved from the Danish Integrated Database for Labour Market Research. ${ }^{29}$

\section{Cardiovascular events and death}

Outcome events were measured as a composite endpoint comprising new cardiovascular events (MI, heart failure, stroke or transient ischaemic attack) and all-cause mortality. Information on outcomes was collected from baseline (the day we sent the questionnaire) to the last day of follow-up (31 July 2012). The Danish National Patient Register $^{22}$ provided information on cardiovascular events. Vital status (dead or alive) was obtained from the Civil Registration System. ${ }^{11}$

\section{Statistical analysis}

Neither natural thresholds nor clinically-based thresholds are defined for the MCS score, so we divided the participants into quartiles according to their score (first quartile had the lowest score; fourth quartile had the highest score). This categorisation was done to enhance clinical interpretability and to evaluate a possible doseresponse relationship.

In order to address the potential risk of selection bias, we used antidepressant consumption as a proxy for depression and calculated HRs for the association between antidepressant consumption and new cardiovascular events or death for both participants and non-participants.

The association between baseline characteristics and MCS score was assessed using $\chi^{2}$ statistics for categorical variables and analysis of variance for continuous variables, or Kruskal-Wallis tests when the conditions for analysis of variance were not fulfilled.

We calculated the event-free survival time as the time from 3 months after MI (baseline evaluation of mental health status) to the first cardiovascular event or death. If no event or death occurred, the participant was censored on 31 July 2012. Two persons emigrated during the time of follow-up, and they were censored on the day of their emigration. Owing to the use of nationwide registers, we had complete follow-up of all participants.

The unadjusted association between mental health status and new cardiovascular events or death was presented graphically with Kaplan-Meier curves. The cumulative incidence 3 years after MI was estimated using the cumulative hazards function, and identical incidence was tested using the log-rank test.

The risk of cardiovascular events or death associated with mental health status was compared using the Cox proportional hazards regression model. The covariates for the multivariate model (age, sex, cohabitation status, education, labour market status, cardiac disease severity, history of stroke, diabetes mellitus, heart failure, secondary prophylactic medication, smoking status, physical activity, depression and anxiety) were chosen on the basis of previous studies. To check for multicollinearity 
between depression/anxiety symptoms and mental health status, we calculated the variance inflation factor, which was 1.5 . Values above 10 indicate multicollinearity. ${ }^{30}$ We evaluated whether the HRs of mental health status following MI varied by subgroups by testing for interaction using the Wald test in an age-adjusted model, and the results are presented in a forest plot. Too few outcome events were available to test for interaction in quartiles, so we tested it in a dichotomised (median cut) model. We excluded variables with less than five events in a subgroup.

Finally, we calculated HRs for the association between each of the mental health status items (continuous; per one-point lower item score) and the outcome.

No variable had more than $0.3 \%$ missing data, except body mass index (for which $2.5 \%$ data were missing) and education (for which $3.3 \%$ data were missing), and analyses were performed on complete data only. $\mathrm{p}<0.05$ was considered statistically significant.

\section{RESULTS}

\section{Participant characteristics}

Among a total of 1288 eligible patients with first-time MI, 880 (68.3\%) completed SF-12, and the mean MCS score was 44.9 (SD 11.5). Non-participants were more often women, older and had fewer socioeconomic resources and more comorbid conditions than participants (see online supplementary table A). The estimates of the association between antidepressant consumption and new cardiovascular events or death in participants, HR 1.55 (95\% CI 1.12 to 2.14) and in non-participants, HR 1.46 (1.01 to 2.10), were similar. Compared to participants with a higher mental health status, the participants with the lowest mental health status (first quartile, table 1) were impaired in a range of variables; for example, symptoms of depression and anxiety, cardiac disease severity, comorbidity, socioeconomic resources and health behaviour.

\section{Cumulative incidence}

A total of 277 outcomes (230 new cardiovascular events and 47 deaths) occurred during 1940 person-years of follow-up (median 2.6 years, SD 1.0). The Kaplan-Meier curves (figure 1) show that the unadjusted risk of a cardiovascular event or death increased with decreasing mental health status. During the 3 years after MI, the cumulative incidence of the composite endpoint was $47.5 \%$ (95\% CI $40.9 \%$ to $54.5 \%$ ) for persons in the first quartile, $37.0 \%$ (30.9\% to $43.9 \%)$ in the second quartile, $29.1 \%(23.5 \%$ to $35.6 \%)$ in the third quartile and $15.0 \%$ $(10.8 \%$ to $20.5 \%)$ in the fourth quartile, $\mathrm{p}<0.001$.

\section{Association between mental health status and new cardiovascular events or death}

The age-adjusted HRs for new cardiovascular events or death in post-MI patients increased with decreasing mental health status $\left(\mathrm{HR}_{3 \mathrm{rd}}\right.$ quartile 2.09 (95\% CI 1.36 to
3.19), $\mathrm{HR}_{2 \text { nd quartile }} 2.67$ (1.77 to 4.03 ), $\mathrm{HR}_{1 \text { st quartile }} 3.53$ (2.36 to 5.27), table 2). Additional adjustment for cardiac disease severity, physical activity, depression and anxiety attenuated the association. In the fully adjusted model, the MI patients with the lowest mental health status had a more than twofold higher risk of new cardiovascular events or death compared to the patients with the highest mental health status (table 2).

We found no statistically significant difference in HRs between any subgroups of MI patients (figure 2).

\section{Exploratory analysis of the six mental health status items}

Table 3 outlines the association between mental health status item scores and subsequent cardiovascular events or death. The items were entered as continuous variables and HRs reflect the risk of new cardiovascular events or death per one point lower item score. The largest HRs were seen for the 'Vitality' item, HR 1.24 (95\% CI 1.09 to 1.42 ), the 'Mental Health' item 1, HR 1.19 (1.04 to 1.35), and the 'Role-Emotional' item 1, HR 1.16 (1.04 to 1.29$)$.

\section{DISCUSSION}

In this population-based cohort study, we found that low mental health status after first-time MI predicted an increased risk of new cardiovascular events or death in a dose-response manner. The association was explained partly by cardiac disease severity, physical activity, depression and anxiety. However, even after adjustments for these variables, patients with the lowest mental health status had a more than twofold higher risk of new cardiovascular events or death compared to those with the highest mental health status.

\section{Strengths and limitations of the study}

The major strengths of this study are its populationbased nature and the homogeneous study population; we invited all patients with first-time MI during 1 year in a well-defined area. Our response rate was reasonably high $(68.3 \%)$, and information on outcome was collected without loss to follow-up. Non-participants tended to have fewer social resources and more comorbid conditions, and hence they resembled the participants with the lowest mental health status. In order to address the potential risk of selection bias, we used antidepressant consumption as a proxy for depressive symptoms, similar to previous studies. ${ }^{31}$ The estimates of the association between antidepressant consumption and new cardiovascular events or death in participants and nonparticipants were similar. Thus, bias due to selection of study participants seems to be an unlikely explanation for our findings.

Information on MI was registered prospectively and did not rely on the participants' or the relatives' memory. The MI diagnosis in the Danish National Patient Register was based on the current European Society of Cardiology criteria for MI, coded by the 
Table 1 Baseline characteristics of 880 patients with first-time myocardial infarction in 2009 by quartiles ${ }^{*}$ of mental health status (Mental Component Summary score from the Short-Form 12 V.2)

\begin{tabular}{|c|c|c|c|c|c|}
\hline \multirow[b]{2}{*}{ Variablet } & \multicolumn{5}{|c|}{ Baseline MCS Score } \\
\hline & $\begin{array}{l}\text { First quartile } \\
\mathrm{n}=220\end{array}$ & $\begin{array}{l}\text { Second quartile } \\
n=220\end{array}$ & $\begin{array}{l}\text { Third quartile } \\
\mathrm{n}=220\end{array}$ & $\begin{array}{l}\text { Fourth quartile } \\
n=220\end{array}$ & $\begin{array}{l}\mathbf{p} \\
\text { Value }\end{array}$ \\
\hline \multicolumn{6}{|l|}{ Self-reported health $\ddagger$} \\
\hline $\begin{array}{l}\text { Mental health status (MCS } \\
\text { score)§, mean (range) }\end{array}$ & $28.8(11.1-37.2)$ & $42.2(37.2-47.0)$ & $51.0(47.0-54.5)$ & $57.7(54.5-60.8)$ & $<0.001$ \\
\hline HADS-A/D $\geq 8$, No. $(\%)$ & $152(69.7)$ & $79(36.07)$ & $22(10.0)$ & $2(0.91)$ & $<0.001$ \\
\hline \multicolumn{6}{|c|}{ Sociodemographic characteristics } \\
\hline Age, years, mean (SD) & $68.9(12.4)$ & $68.4(12.3)$ & $65.6(11.2)$ & $64.5(10.0)$ & $<0.001$ \\
\hline Male sex, $\mathrm{N}(\%)$ & $120(54.6)$ & $138(62.7)$ & $177(80.5)$ & $173(78.6)$ & $<0.001$ \\
\hline $\begin{array}{l}\text { Cohabitation status, living } \\
\text { alone, } \mathrm{N}(\%) \uparrow\end{array}$ & $94(42.7)$ & $82(37.3)$ & $55(25.0)$ & $47(21.4)$ & $<0.001$ \\
\hline \multicolumn{6}{|l|}{ Education, $\mathrm{N}(\%) \rrbracket$} \\
\hline$<10$ years & $114(53.3)$ & $105(50.2)$ & $85(39.7)$ & $76(35.5)$ & \\
\hline $10-12$ years & 76 (35.5) & $81(38.8)$ & $99(46.3)$ & $101(47.2)$ & \\
\hline$>12$ years & $24(11.2)$ & $23(11.0)$ & $30(14.0)$ & $37(173)$ & 0.004 \\
\hline \multicolumn{6}{|l|}{ Labour market status, $\mathrm{N}(\%) \emptyset$} \\
\hline Working & $50(22.7)$ & $70(31.8)$ & $99(45.0)$ & $103(46.8)$ & \\
\hline Pension & $136(61.8)$ & $123(55.9)$ & $105(47.7)$ & $107(48.6)$ & \\
\hline Out of the work force & $34(15.5)$ & 27 (12.3) & $16(7.3)$ & $10(4.6)$ & $<0.001$ \\
\hline \multicolumn{6}{|l|}{ Health status $\ddagger$} \\
\hline Body mass index, mean (SD) & $26.5(5.1)$ & $26.3(4.8)$ & $26.8(4.5)$ & $26.9(4.5)$ & 0.626 \\
\hline \multicolumn{6}{|l|}{ Comorbid conditions, $\mathrm{N}(\%)^{\star \star}$} \\
\hline Hypertension†† & $88(40.0)$ & $75(34.1)$ & $54(24.6)$ & $54(24.6)$ & $<0.001$ \\
\hline Stroke & $21(9.6)$ & $16(7.3)$ & 7 (3.2) & $5(2.3)$ & 0.002 \\
\hline $\mathrm{TCl}$ & $12(5.5)$ & $3(1.4)$ & $3(1.4)$ & $10(4.6)$ & 0.021 \\
\hline Revascularisation & $37(16.8)$ & $16(7.3)$ & $12(5.5)$ & $15(6.8)$ & $<0.001$ \\
\hline Heart failure & $16(7.3)$ & $4(1.8)$ & $4(1.8)$ & $4(1.8)$ & $<0.001$ \\
\hline Diabetes mellitus & $51(23.2)$ & $38(17.3)$ & 24 (10.9) & $21(9.6)$ & $<0.001$ \\
\hline 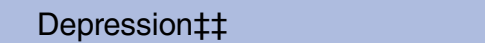 & $44(20.0)$ & $21(9.6)$ & $11(5.0)$ & $9(4.1)$ & $<0.001$ \\
\hline \multicolumn{6}{|l|}{ Cardiac disease severity $\ddagger$} \\
\hline $\begin{array}{l}\text { MRC dyspnoea score } \geq 3 \\
\text { N (\%) }\end{array}$ & $110(50.2)$ & $45(20.5)$ & $21(9.6)$ & $3(1.4)$ & $<0.001$ \\
\hline \multicolumn{6}{|l|}{ Medication use, N (\%)‡ } \\
\hline Aspirin & $166(75.5)$ & $168(76.4)$ & $173(78.6)$ & $186(84.6)$ & 0.086 \\
\hline Clopidogrel & 159 (72.3) & $164(74.6)$ & $173(78.6)$ & $184(83.6)$ & 0.025 \\
\hline$\beta$-Blocker & $174(79.1)$ & $181(82.3)$ & $178(80.9)$ & $180(81.8)$ & 0.837 \\
\hline Statin & $169(76.8)$ & $184(83.6)$ & $190(86.4)$ & $195(88.6)$ & 0.005 \\
\hline $\begin{array}{l}\text { ACE-inhibitors/AT-II-receptor } \\
\text { block }\end{array}$ & $111(50.5)$ & $111(50.5)$ & $107(48.6)$ & $100(45.5)$ & 0.689 \\
\hline $\begin{array}{l}\text { Furosemide/aldosterone } \\
\text { antagonist }\end{array}$ & $93(42.3)$ & $64(29.1)$ & $35(15.9)$ & $27(12.3)$ & $<0.001$ \\
\hline Antidepressants & $53(24.1)$ & $24(10.9)$ & $9(4.1)$ & $8(3.6)$ & $<0.001$ \\
\hline $\begin{array}{l}\text { Secondary prophylactic } \\
\text { medication }\end{array}$ & $146(66.4)$ & $160(72.7)$ & $162(73.6)$ & $166(75.5)$ & 0.163 \\
\hline \multicolumn{6}{|l|}{ Potential behavioural mediatorsł } \\
\hline $\begin{array}{l}\text { Alcohol consumption >14/21 } \\
\text { units/week, N (\%) }\end{array}$ & $8(3.6)$ & $12(5.5)$ & $8(3.6)$ & $14(6.4)$ & 0.438 \\
\hline \multicolumn{6}{|l|}{ Smoking status, $N(\%)$} \\
\hline Current & $54(24.8)$ & $49(22.4)$ & $44(20.0)$ & $30(13.6)$ & \\
\hline Past & $124(56.9)$ & $122(55.7)$ & $121(55.0)$ & $128(58.2)$ & \\
\hline Never & $40(18.4)$ & $48(21.9)$ & $55(25.0)$ & $62(28.2)$ & 0.048 \\
\hline $\begin{array}{l}\text { Intake of fruit and vegetables } \geq 3 \\
\text { portions/d, N (\%) }\end{array}$ & $69(31.4)$ & $75(34.1)$ & $86(39.1)$ & $100(45.5)$ & 0.013 \\
\hline Intake of fish $\geq 3$ times/day, $\mathrm{N}$ (\%) & $61(27.7)$ & $78(35.5)$ & $93(42.5)$ & $96(43.8)$ & 0.001 \\
\hline $\begin{array}{l}\text { Intake of fish oil supplement, } \\
\mathrm{N}(\%)\end{array}$ & 57 (25.9) & $50(22.7)$ & 75 (34.1) & 69 (31.4) & 0.035 \\
\hline
\end{tabular}


Table 1 Continued

\begin{tabular}{|c|c|c|c|c|c|}
\hline \multirow[b]{2}{*}{ Variable } & \multicolumn{5}{|c|}{ Baseline MCS Score } \\
\hline & $\begin{array}{l}\text { First quartile } \\
\mathrm{n}=220\end{array}$ & $\begin{array}{l}\text { Second quartile } \\
n=220\end{array}$ & $\begin{array}{l}\text { Third quartile } \\
\mathrm{n}=220\end{array}$ & $\begin{array}{l}\text { Fourth quartile } \\
\mathrm{n}=220\end{array}$ & $\begin{array}{l}\mathbf{p} \\
\text { Value } \\
\end{array}$ \\
\hline $\begin{array}{l}\text { Physical activity, days/week, } \\
\text { mean (SD) }\end{array}$ & $3.6(2.8)$ & $5.1(2.3)$ & $5.3(2.1)$ & $5.7(1.8)$ & $<0.001$ \\
\hline $\begin{array}{l}\text { Participation in phase two cardiac } \\
\text { rehabilitation } \ddagger\end{array}$ & $110(50.2)$ & $119(54.1)$ & $144(65.5)$ & $142(64.8)$ & 0.001 \\
\hline \multicolumn{6}{|c|}{ 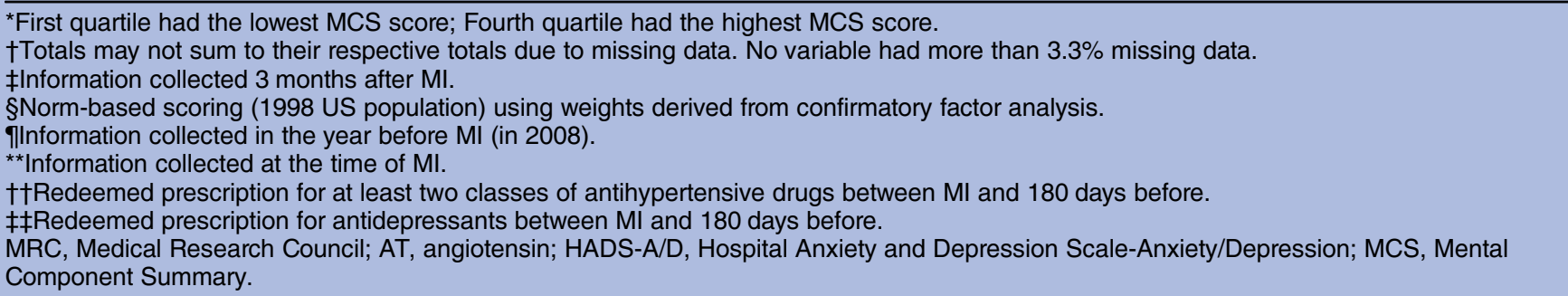 } \\
\hline
\end{tabular}

physician in charge of the discharge, and the information is known to have a high sensitivity (90\%) and specificity $(92 \%){ }^{10}$ The specificity was even higher in our study because we confirmed the MI diagnosis by reviewing the discharge summaries, ${ }^{9}$ and this reduced the risk of information bias. We also reduced the risk of information bias by using previously translated and validated scales, pilot testing the questionnaire among MI patients, and using high-quality register data.

We used a new algorithm for the calculation of the MCS score from the SF-12 V.2 using weights constructed by oblique confirmatory factor analysis, which allows the physical and mental component summary score to be correlated. Fleishman et $a l^{15}$ developed this new scoring algorithm due to the controversy regarding the traditional scoring algorithm. ${ }^{32-34}$ The traditional scoring algorithm forces mental and physical health to be uncorrelated. Consequently, when physical scores are well below the mean and mental scores somewhat less below the mean, as is often the case in patients with physical illness, this scoring method will result in an artefactual migration of the MCS score towards the mean. ${ }^{32}$ In subanalyses, we estimated HRs based on traditionally computed MCS scores (see online supplementary table B). As expected, they were smaller compared to the HRs based on MCS scores computed with the new scoring algorithm. We evaluated mental health status 3 months after MI, allowing mental health to reach a more stable level after this major life event.

A diagnosis of depression or anxiety should ideally be based on a diagnostic interview. Since a previous study has estimated the sensitivity of the HADS-D $\geq 8$ for identification of depression to be $65 \%$ in MI patients, ${ }^{19}$ a substantial number of participants with depression may
Figure 1 Kaplan-Meier curves by quartiles of mental health status (Mental Component Summary score from the Short-Form 12 V.2).

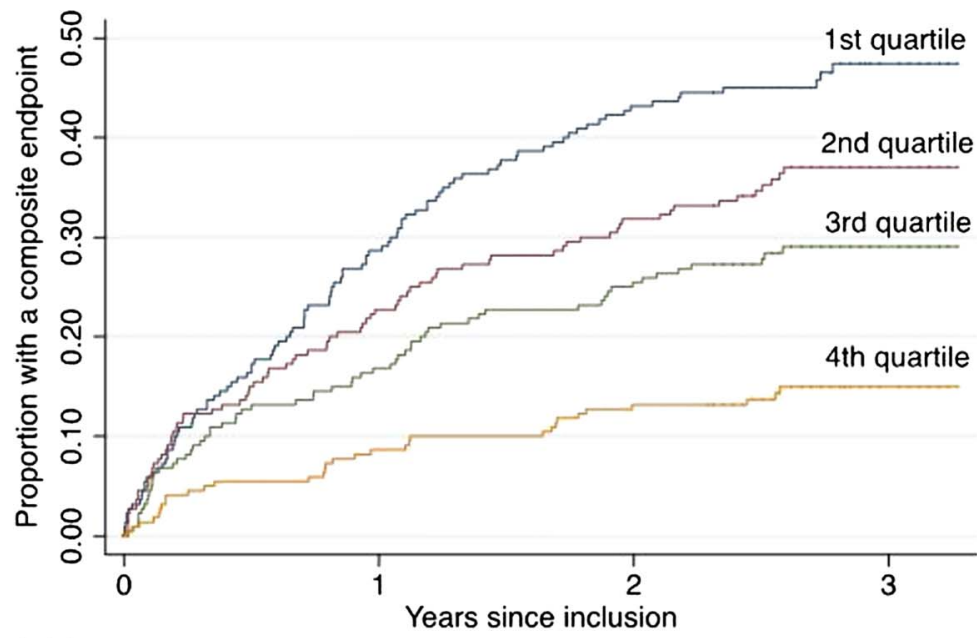

Number at risk 1 st quartile 220 2nd quartile 220 3rd quartile 220 
Table 2 Association between mental health status (Mental Component Summary score from the Short-Form 12 V.2) and subsequent cardiovascular events or death, with sequential adjustment for potential confounders

\begin{tabular}{|c|c|c|c|c|}
\hline \multirow[b]{2}{*}{ Adjusted variables* } & \multicolumn{4}{|l|}{ HR $(95 \% \mathrm{Cl})$} \\
\hline & $\begin{array}{l}\text { First quartile MCS } \\
(102 / 220) \dagger\end{array}$ & $\begin{array}{l}\text { Second quartile MCS } \\
(80 / 220) \dagger\end{array}$ & $\begin{array}{l}\text { Third quartile MCS } \\
(63 / 220) \dagger\end{array}$ & $\begin{array}{l}\text { Fourth quartile } \\
\text { MCS }(32 / 220) \dagger\end{array}$ \\
\hline Age & 3.53 (2.36 to 5.27$)$ & 2.67 (1.77 to 4.03$)$ & 2.09 (1.36 to 3.19$)$ & 1 (reference) \\
\hline $\begin{array}{l}\text { Sociodemographic } \\
\text { characteristics } \ddagger\end{array}$ & 3.56 (2.35 to 5.38$)$ & 2.57 (1.69 to 3.92$)$ & $2.06(1.34$ to 3.16$)$ & 1 (reference) \\
\hline MRC dyspnoea score $\geq 3$ & 2.74 (1.76 to 4.26$)$ & 2.30 (1.50 to 3.53$)$ & 1.96 (1.27 to 3.00$)$ & 1 (reference) \\
\hline Comorbidity§ & 2.65 (1.70 to 4.13$)$ & $2.29(1.50$ to 3.51$)$ & 1.99 (1.29 to 3.05$)$ & 1 (reference) \\
\hline $\begin{array}{l}\text { Secondary prophylactic } \\
\text { medication }\end{array}$ & 2.77 (1.78 to 4.31$)$ & 2.32 (1.51 to 3.56$)$ & 1.95 (1.27 to2.99) & 1 (reference) \\
\hline Smoking status & $2.76(1.76$ to 4.31$)$ & 2.31 (1.51 to 3.56$)$ & 1.96 (1.27 to 3.01$)$ & 1 (reference) \\
\hline Physical activity & 2.47 (1.56 to 3.91$)$ & 2.25 (1.47 to 3.46$)$ & 1.89 (1.23 to 2.91$)$ & 1 (reference) \\
\hline HADS-A/D score $\geq 8$ & 2.26 (1.37 to 3.73$)$ & 2.15 (1.38 to 3.35$)$ & 1.87 (1.21 to 2.88$)$ & 1 (reference) \\
\hline
\end{tabular}

have been misclassified as not having depression. However, we identified $18.3 \%$ with depression in our population (HADS-D $\geq 8$ ), which is in keeping with the prevalence of post-MI depression identified by structured clinical interviews in other studies $(19.8 \%){ }^{1}$ We found no studies reporting on the sensitivity and specificity of HADS-A in an MI population. However, among acute coronary syndrome patients, a HADS-A $\geq 8$ had a sensitivity of $91 \% .^{21}$ Accordingly, we most likely identified the majority of patients with anxiety. In a sensitivity analysis, we excluded patients with depression or anxiety (HADS-A/D $\geq 8$ ), but this did not weaken the estimates (see online supplementary table $\mathrm{C}$ ).

Schizophrenia and bipolar disorder are known to be associated with a higher risk of mortality, and part of this excess risk is attributable to cardiovascular diseases. $^{35}$ We used a prescription of antipsychotics, between MI and 180 days before, as an approximation of severe mental disorder. Thirteen participants had redeemed such a prescription. To examine how much of the association could be explained by these patients, we excluded this group in a sensitivity analysis (not shown), but this did not weaken the estimates.

Lifestyle behaviour was self-reported, and participants with low mental health status may have been more likely to under-report adverse lifestyle, including physical inactivity. However, participants with low mental health status did in fact report adverse lifestyle in our study, and a study on depression ${ }^{36}$ found no differences when substituting self-reported physical activity with an objective measure of physical fitness.

Information on a range of participant characteristics and the large sample size allowed us to take into account several potential confounders, such as sociodemographic characteristics, cardiac disease severity, comorbidity and behavioural factors. In subanalyses, we adjusted for other potential confounders (body mass index, hypertension, history of depression, antidepressant use, intake of alcohol, fish and fruit, and participation in phase two cardiac rehabilitation), but this did not change the estimates $(\leq 4 \%)$. However, we cannot rule out the possibility of residual confounding.

\section{Comparison with other studies}

Four previous studies ${ }^{5-8}$ have investigated the association between mental health status after MI and prognosis independent of various clinical risk factors, such as disease severity. They used different measures of mental health status (COOP charts, ${ }^{6}$ Quality of Life after MI questionnaire, $^{8}$ the WHO Quality of Life Instrument Abbreviated $^{7}$ and SF-12 $2^{5}$, and they all found an independent association between low mental health status and higher risk of adverse outcomes. Compared with our study, these studies were conducted in modest-sized cohorts $\left(\mathrm{n}=112,{ }^{6} 375,{ }^{8} 145^{7}\right)$ and had short follow-up (4-5 months, ${ }^{6} 18$ months $^{8}$ ); also, mental health status was assessed up to 5 years or more after $\mathrm{MI}^{5} 7$ and included only women ${ }^{7}$ or patients who had an ejection fraction $<30 \%$. $^{5}$ Most importantly, none of these four MI studies took into account important mediators such as depression, anxiety and potential behavioural mediators such as physical activity.

Our study is the first to explore the association between mental health status after MI and new cardiovascular events or death in subgroups, and we identified no factors that modified the risk. However, the sample size was low in some of the subgroups.

Our study is also the first to explore the association between mental health status and cardiovascular events or death on an item level. We found that the 'Vitality' item, the 'Role-Emotional' item 1, and the 'Mental Health' item 1 were significantly associated with adverse 
Figure 2 Association between baseline mental health status (median cut) and subsequent cardiovascular events or death for patients with myocardial infarction and specific characteristics.

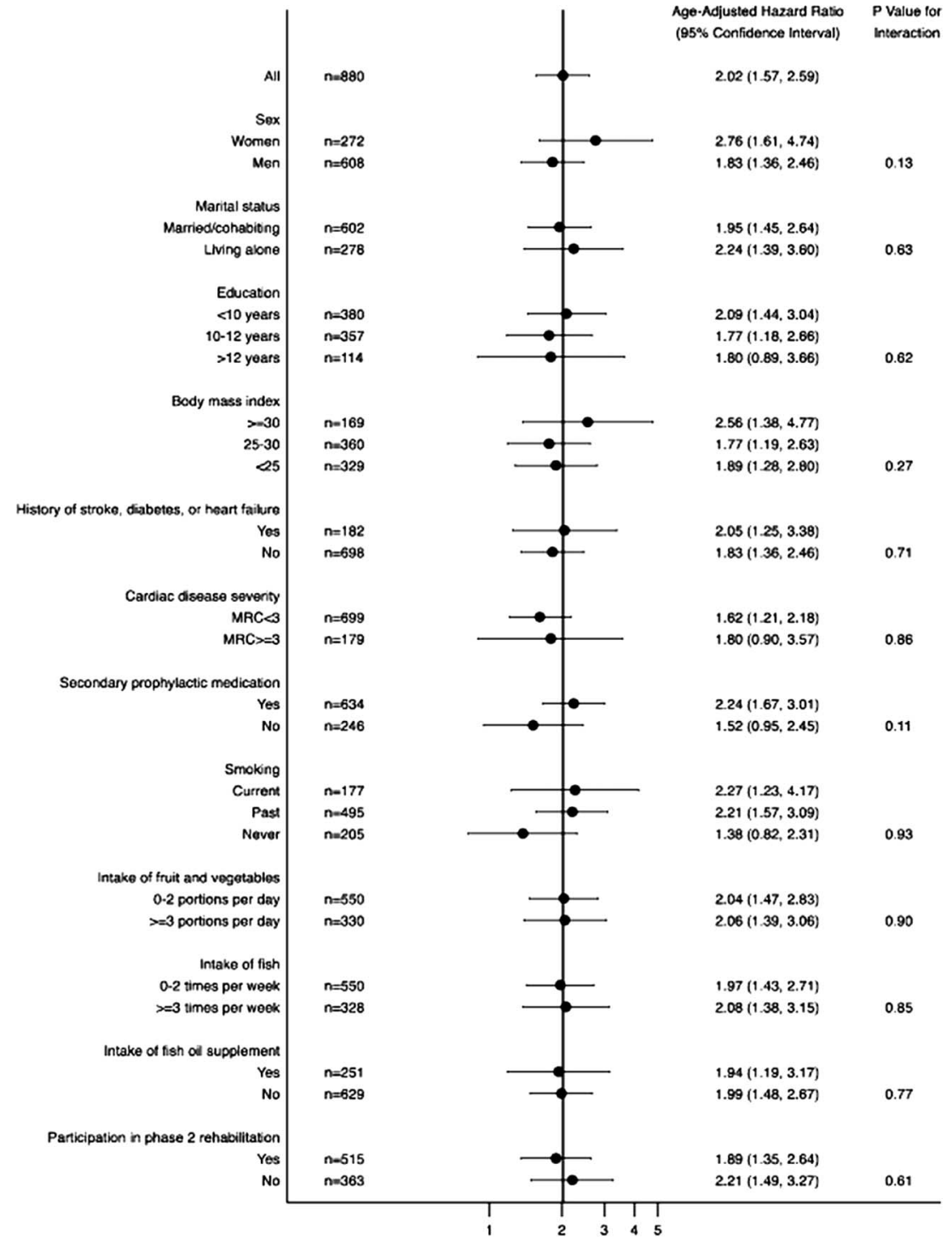

events after adjustments for clinical, sociodemographic, behavioural and other psychological risk factors, whereas the remaining items were not. Our results indicate that these items are the most important for the association between mental health status (MCS score) and adverse events. Yet, it is important to keep in mind that the items have different weights and that the physical items are also included when computing the MCS score. $^{1215}$

\section{Implications for clinicians}

In addition to psychological, social and functional impairment, clinicians should be aware that low mental health status following MI is associated with an increased risk of new cardiovascular events and death. Our results underline the importance of always considering and prioritising mental health issues in postMI patients. In this study, we identified low mental health status after MI to be a significant risk factor for poor prognosis, independent of clinical, sociodemographic, behavioural and other psychological risk factors. In other words, mental health status has incremental value in the identification of patients at elevated risk for adverse outcome. Adding mental health status measurement to our present risk factor armamentarium could help clinicians to distinguish between groups of patients with a very low versus a very high risk of adverse outcome, and thereby help identify vulnerable patients in need of optimised care. However, we do not know whether measurement of mental health status and improved knowledge of prognosis will translate into better outcomes for our patients. This is an important focus for future research in this field.

\section{Possible explanations and future research}

This study suggests that mental health status may capture prognostic aspects of mental health that are not captured by measures of depression and anxiety. Further research is needed to clarify more specifically what aspects of mental health are at play. 


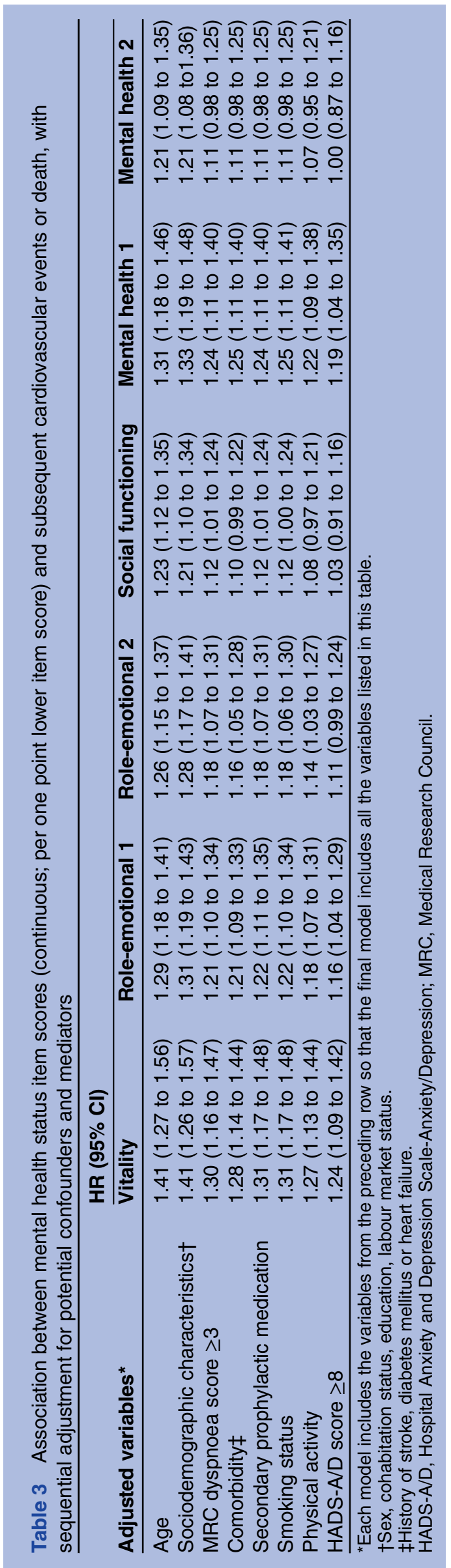

The underlying explanation for the association between mental health status after MI and new cardiovascular events or death remains unclear. Our study evaluated cardiac disease severity, behavioural factors and treatment strategies concurrently with mental health status. Therefore, we cannot determine whether these factors were the cause or the result of the mental health status. We were unable to assess whether the association was explained by biological mechanisms (such as heart rate variability, platelet function or inflammatory mechanisms) since we had no information on these biological variables. Future studies should incorporate such biological variables. ${ }^{37}$

Further research is also needed to identify interventions that can improve both mental health status and prognosis in MI patients. Murphy et $a l^{88}$ examined the effectiveness of a complex intervention designed to improve outcomes, including mental health status (measured with SF-12) for patients with coronary heart disease in a cluster randomised controlled trial. The intervention was "tailored care plans for practices (practice based training in prescribing and behaviour change, administrative support, quarterly newsletter) and tailored care plans for patients (motivational interviewing, goal identification, and target setting for lifestyle change)." ${ }^{38}$ They found that admissions to the hospital were significantly reduced after an intensive 18-month intervention to improve outcomes for patients with coronary heart disease, but there was no change in mental health status. It was not stated how they computed the MCS score, but they probably used the traditional scoring algorithm as the study was conducted prior to Fleishman's publication. ${ }^{15}$ Hence, artefactual migration of the MCS score towards the mean in these physically ill participants may, at least in part, explain the lack of association.

\section{CONCLUSION}

We found that low mental health status following MI was associated with an increased risk of new cardiovascular events or death. The association was explained partly by cardiac disease severity, physical activity, depression and anxiety, but low mental health status remained an independent prognostic risk factor. Further research is needed to disentangle the pathways that link mental health status following MI to prognosis and, in continuation hereof, to identify interventions that can improve mental health status and prognosis.

Contributors TJN, KKL, MV, BC and KSC conceived the study idea and designed the study. KKL collected the data. TJN, KKL, MV, BC and KSC reviewed the literature. TJN, KKL, MV, BC and KSC directed the analyses, which were carried out by TJN. All authors participated in the discussion and interpretation of the results. TJN organised the writing and wrote the initial drafts. All authors critically revised the manuscript for intellectual content and approved the final version. TJN is the guarantor.

Funding The study was supported by the Danish Independent Research Council (grant number 12-126032), the Tryg Foundation (grant number 7844-07), the Danish Health Insurance Foundation (grant number 2010B013) 
and the Lundbeck Foundation. None of the funding sources had a role in the design, conduct, analysis or reporting of the study.

Competing interests TJN had financial support from the Danish Independent Research Council for the submitted work.

Provenance and peer review Not commissioned; externally peer reviewed.

Data sharing statement No additional data are available.

Open Access This is an Open Access article distributed in accordance with the Creative Commons Attribution Non Commercial (CC BY-NC 3.0) license, which permits others to distribute, remix, adapt, build upon this work noncommercially, and license their derivative works on different terms, provided the original work is properly cited and the use is non-commercial. See: http:// creativecommons.org/licenses/by-nc/3.0/

\section{REFERENCES}

1. Thombs BD, Bass EB, Ford DE, et al. Prevalence of depression in survivors of acute myocardial infarction-review of the evidence. $J$ Gen Intern Med 2006;21:2130-8.

2. Roest AM, Martens EJ, Denollet J, et al. Prognostic association of anxiety post myocardial infarction with mortality and new cardiac events: a meta-analysis. Psychosom Med 2010;72:563-9.

3. Crilley JG, Farrer M. Impact of first myocardial infarction on self-perceived health status. QJM 2001;94:13-18

4. Meijer A, Conradi HJ, Bos EH, et al. Prognostic association of depression following myocardial infarction with mortality and cardiovascular events: a meta-analysis of 25 years of research. Gen Hosp Psychiatry 2011;33:203-16.

5. Piotrowicz K, Noyes K, Lyness JM, et al. Physical functioning and mental well-being in association with health outcome in patients enrolled in the Multicenter Automatic Defibrillator Implantation Trial II. Eur Heart J 2007;28:601-7.

6. Nelson EC, Ferreira PL, Cleary PD, et al. Do patients' health status reports predict future hospital stays for patients with an acute myocardial infarction?. Fam Pract Res J 1994;14:119-26.

7. Norekval TM, Fridlund B, Rokne B, et al. Patient-reported outcomes as predictors of 10-year survival in women after acute myocardial infarction. Health Qual Life Outcomes 2010;8:140.

8. Lim LL, Johnson NA, O'Connell RL, et al. Quality of life and later adverse health outcomes in patients with suspected heart attack. Aust N Z J Public Health 1998;22:540-6.

9. Larsen KK, Vestergaard M, Sondergaard J, et al. Rehabilitation status three months after first-time myocardial infarction. Scand J Prim Health Care 2011;29:210-15.

10. Joensen AM, Jensen MK, Overvad K, et al. Predictive values of acute coronary syndrome discharge diagnoses differed in the Danish National Patient Registry. J Clin Epidemiol 2009;62:188-94.

11. Pedersen CB. The Danish Civil Registration System. Scand J Public Health 2011;39(7 Suppl):22-5

12. Kosinski M, Ware JE, Turner-Bowker DM, et al. User's manual for the SF-12v2 health survey: with a supplement documenting the SF-12® health survey. Lincoln, RI: QualityMetric incorporated, 2007.

13. Ware JE, Kosinski M, Keller SD. A 12-item short-form health survey - construction of scales and preliminary tests of reliability and validity. Med Care 1996;34.

14. Gandek B, Ware JE, Aaronson NK, et al. Cross-validation of item selection and scoring for the SF-12 Health Survey in nine countries: results from the IQOLA Project. International Quality of Life Assessment. J Clin Epidemiol 1998;51:1171-8.

15. Fleishman JA, Selim AJ, Kazis LE. Deriving SF-12v2 physical and mental health summary scores: a comparison of different scoring algorithms. Qual Life Res 2010;19:231-41.

16. Zigmond AS, Snaith RP. The Hospital Anxiety and Depression Scale. Acta Psychiatr Scand 1983;67:361-70.
17. Johnston M, Pollard B, Hennessey P. Construct validation of the hospital anxiety and depression scale with clinical populations. J Psychosom Res 2000;48:579-88.

18. Martin C, Lewin R, Thompson D. A confirmatory factor analysis of the Hospital Anxiety and Depression Scale in coronary care patients following acute myocardial infarction. Psychiatry Res 2003;120:85-94.

19. Thombs BD, Magyar-Russell G, Bass EB, et al. Performance characteristics of depression screening instruments in survivors of acute myocardial infarction: review of the evidence. Psychosomatics 2007;48:185-94.

20. Bjelland I, Dahl AA, Haug TT, et al. The validity of the Hospital Anxiety and Depression Scale. An updated literature review. J Psychosom Res 2002;52:69-77.

21. Frasure-Smith N, Lesperance F. Depression and anxiety as predictors of 2-year cardiac events in patients with stable coronary artery disease. Arch Gen Psychiatry 2008;65:62-71.

22. Andersen TF, Madsen M, Jorgensen J, et al. The Danish National Hospital Register. A valuable source of data for modern health sciences. Dan Med Bull 1999;46:263-8.

23. Carstensen B, Kristensen JK, Marcussen MM, et al. The National Diabetes Register. Scand J Public Health 2011;39:58-61.

24. Johannesdottir SA, Horvath-Puho E, Ehrenstein V, et al. Existing data sources for clinical epidemiology: the Danish National Database of Reimbursed Prescriptions. Clin Epidemiol 2012;4:303-13.

25. Fletcher C. Standardized questionaires on respiratory symptoms. A statement prepared for, and approved by, the Medical Research Council's Committee on the aetiology of chronic bronchitis. $\mathrm{Br} \mathrm{Med} \mathrm{J}$ 1960;2:1665.

26. Vestbo J, Knudsen KM, Rasmussen FV. Should we Continue using Questionnaires on Breathlessness in Epidemiologic Surveys. Am Rev Respir Dis 1988;137:1114-8.

27. Ades PA. Cardiac rehabilitation and secondary prevention of coronary heart disease. N Engl J Med 2001;345:892-902.

28. Giannuzzi P, Saner H, Bjornstad H, et al. Secondary prevention through cardiac rehabilitation: position paper of the Working Group on Cardiac Rehabilitation and Exercise Physiology of the European Society of Cardiology. Eur Heart J 2003;24:1273-8.

29. Statistics Denmark. IDA: an integrated database for labour market research: main report. Copenhagen, Denmark: Statistics Denmark, 1991.

30. Wetherill G, Duncombe P, Kenward M. Regression analysis with applications. London: Capman and Hall, 1986.

31. Barnett K, Mercer SW, Norbury M, et al. Epidemiology of multimorbidity and implications for health care, research, and medical education: a cross-sectional study. Lancet 2012;380:37-43.

32. Farivar SS, Cunningham WE, Hays RD. Correlated physical and mental health summary scores for the SF-36 and SF-12 Health Survey, V.I. Health Qual Life Outcomes 2007;5:54.

33. Pelle AJ, Kupper N, Mols F, et al. What is the use? Application of the short form (SF) questionnaires for the evaluation of treatment effects. Qual Life Res 2012. doi:10.1007/s11136-012-0266-8

34. Hann M, Reeves D. The SF-36 scales are not accurately summarised by independent physical and mental component scores. Qual Life Res 2008;17:413-23.

35. Hoang U, Stewart R, Goldacre MJ. Mortality after hospital discharge for people with schizophrenia or bipolar disorder: retrospective study of linked English hospital episode statistics, 1999-2006. BMJ 2011;343:d5422.

36. Whooley MA, De Jonge $P$, Vittinghoff $E$, et al. Depressive symptoms, health behaviors, and risk of cardiovascular events in patients with coronary heart disease. JAMA2008;300:2379-88.

37. Rumsfeld JS, Ho PM. Depression and cardiovascular disease: a call for recognition. Circulation 2005;111:250-3.

38. Murphy AW, Cupples ME, Smith SM, et al. Effect of tailored practice and patient care plans on secondary prevention of heart disease in general practice: cluster randomised controlled trial. BMJ 2009;339: b4220. 